\title{
ShiF acts as an auxiliary factor of aerobactin secretion in meningitis Escherichia coli strain S88
}

\author{
Mathieu Genuini ${ }^{1,2}$, Philippe Bidet ${ }^{1,2}$, Jean-François Benoist ${ }^{3,4}$, Dimitri Schlemmer ${ }^{3}$, Chloé Lemaitre ${ }^{1,2}$, \\ André Birgy ${ }^{1,2}$ and Stéphane Bonacorsi ${ }^{1,2^{*}}$
}

\begin{abstract}
Background: The neonatal meningitis E. coli (NMEC) strain S88 carries a ColV plasmid named pS88 which is involved in meningeal virulence. Transcriptional analysis of pS88 in human serum revealed a strong upregulation of an ORF of unknown function: shiF, which is adjacent to the operon encoding the siderophore aerobactin. The aim of this work is to investigate the role of shiF in aerobactin production in strain S88.

Results: Study of the prevalence of shiF and aerobactin operon in a collection of 100 extra-intestinal pathogenic $E$. coli strains (EXPEC) and 50 whole genome-sequenced E. coli strains revealed the colocalization of these two genes for $98 \%$ of the aerobactin positive strains. We used Datsenko and Wanner's method to delete shiF in two S88 mutants. A cross-feeding assay showed that these mutants were able to excrete aerobactin meaning that shif is dispensable for aerobactin excretion. Our growth assays revealed that the shif-deleted mutants grew significantly slower than the wild-type strain $\mathrm{S} 88$ in iron-depleted medium with a decrease of maximum growth rates of 23 and $28 \%(p<0.05)$. Using Liquid Chromatography-Mass Spectrometry, we identified and quantified siderophores in the supernatants of S88 and its shiF deleted mutants after growth in iron-depleted medium and found that these mutants secreted significantly less aerobactin than S88 $(-52 \%$ and $-49 \%, p<0.001)$.

Conclusions: ShiF is physically and functionally linked to aerobactin. It provides an advantage to E. coli 588 under iron-limiting conditions by increasing aerobactin secretion and may thus act as an auxiliary virulence factor.
\end{abstract}

Keywords: Escherichia coli, Siderophore, Aerobactin, ShiF

\section{Background}

The neonatal meningitis E. coli (NMEC) strain S88 carries a ColV plasmid named pS88 which is involved in meningeal virulence $[1,2]$. We have previously performed a transcriptional analysis of pS88 in human serum to improve comprehension of virulence determinants of pS88 in neonatal meningitis. This study revealed a strong transcription upregulation for all iron uptake systems, including the well-known siderophores salmochelin and aerobactin, but also for two genes with unknown function: $s s b L$ and $s h i F$ [3]. We have previously described the role of $s s b L$, the most

\footnotetext{
* Correspondence: stephane.bonacorsi@rdb.aphp.fr

${ }^{1}$ University Paris Diderot, Sorbonne Paris Cité, IAME, F-75018 Paris, France

${ }^{2}$ Service de microbiologie, Hôpital Robert-Debré, AP-HP, 48 Bd Sérurier,

75019 Paris, France

Full list of author information is available at the end of the article
}

strongly upregulated gene in these conditions and showed that it acts as an auxiliary virulence factor by boosting the catecholate and phenolate siderophores (yersiniabactin and salmochelin) production through the shikimate pathway [4].

In this work, we focused on shiF which was first described in a pathogenic island named SHI-2 in a Shigella flexneri strain [5]. In this strain, as in S88, shiF is adjacent to aerobactin operon and shares the same Fur box consensus sequence and promoting region [3]. Its amino-acid sequence shares a strong (86\%) similarity with a membrane protein belonging to the Major Facilitator Superfamily (MFS) but shiF as yet no known function.

The aim of this work is to investigate the role of shiF in aerobactin production in strain S88 in order to get 
insight the iron metabolism of extra-intestinal pathogenic E. coli strains (ExPEC).

\section{Results}

The shiF gene is strongly linked to aerobactin operon in human ExPEC strains

In order to study the physical link between shiF and the aerobactin operon, we performed in silico analysis of 50 whole genome sequenced E. coli strains and PCR analysis of 100 ExPEC strains from our collection. Aerobactin operon was found in 17 of the 50 sequenced strains and 83 of our 100 strains ( 34 and $83 \%$ respectively). For 98 of the 100 aerobactin positive strains, we noticed the copresence and the colocalization of shiF and iucA. Commensal strain ED1a is one of the two exceptions. This strain possesses chromosomal aerobactin operon adjacent to shiF but shiF is disrupted by a $10 \mathrm{~kb}$ insert. The other exception strain was isolated from the cerebral spinal fluid of a newborn with iatrogenic meningitis after rachianasthesia. For all the strains lacking aerobactin operon $(n=33+17)$ shiF was also missing.

\section{Presence of shiF is dispensable for secretion of aerobactin siderophore}

In our cross-feeding experiments, comparison of the strain LG1522 growth halo observed around different strains showed no difference between S88 and its shiF-

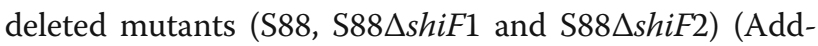
itional file 1: Figure S1). The iatrogenic NMEC strain which contains aerobactin operon but not $\operatorname{shiF}$ was also able to secrete aerobactin. Conversely, no growth of LG1522 was observed in strains S88 4 pS88 and ED1a, showing their inability to secrete aerobactin.

\section{Deletion of shiF induces a slower growth in iron-depleted medium}

We wanted to perform a quantitative assay to analyze more precisely the potential role of shiF during growth.

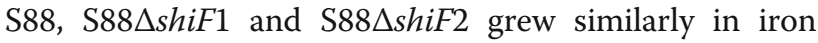
supplemented MM9 and in lysogeny broth (LB) meaning that the lack of shiF has no effect on growth of S88 in iron-rich medium.

In iron-depleted MM9 medium, maximum growth rates showed a significant decrease for both mutants $(23$ and $28 \%$ respectively; $p<0.05)$ and maximum cell densities were $12 \%$ less $(p<0.01)$ when compared to the wild type strain S88. No lag between the start of growth was observed between these strains. Growth curves are presented in Fig. 1.

\section{Deletion of shiF decreases aerobactin secretion}

We investigated siderophores production by Liquid Chromatography-Mass Spectrometry (LC-MS/MS). We identified specific transitions of aerobactin, enterobactin, salmochelin S1 and S2 and yersiniabactin and confirmed their identity by isotope labeling (Fig. 2).We compared siderophores concentration in culture supernatants of strains grown for $14 \mathrm{~h}$ in iron-depleted MM9 medium. Quantification results are shown in Fig. 3. Both shiF-deleted strains secreted significantly less aerobactin in their supernatants compared to wild type strain S88 (-52\% and $-49 \%, p<0.001)$. There was no significant difference among the strains for production of other siderophores. Intracellular quantification of aerobactin from bacterial pellets showed no significant difference between wild type strain and both shiF-deleted mutants (data not shown).

Analyses of strain ED1a showed its ability to produce enterobactin and yersiniabactin but we couldn't detect any production of aerobactin in accordance with crossfeeding experiment (data not shown).

\section{Discussion}

Iron uptake is essential for E. coli growth in urine and blood and siderophores have been shown to play a major role in extra-intestinal virulence [6].

We have previously shown that the transcription of shiF, a plasmid located gene contiguous to aerobactin operon in S88 strain, was strongly upregulated during growth in iron depleted environment [3]. As in S88, the sequence analysis of $E$. coli genomes available on data bank revealed a physical link between shiF and the aerobactin operon. Here we confirmed this strong physical link in our collection of 100 ExPEC strains. ShiF was present and colocalized for $98 \%$ of the strains harboring the plasmidic or chromosomic located aerobactin operon. This systematic colocalization and inducibility under low iron condition suggest for shiF a role in aerobactin expression and the coacquisition of shiF and aerobactin operon was probably guided by a selective advantage.

There are very few data on the role of shiF. In a previous work, a cross feeding experiment showed that an $E$. coli strain deleted for shiF was able to feed LG1522 which is aerobactin dependent to grow in iron-depleted environment [7]. We did the same assay with our shiFdeleted S88 mutants and found the same results. This qualitative experiment led us to conclude that shiF is not essential for aerobactin production and/or excretion. However this does not exclude an auxiliary role of shiF and we used a quantitative approach to specify the function of shiF.

Our growth assays showed that shiF was potentially involved in iron metabolism. The shiF mutants grew with a significant lower maximum growth rate in an ironlimited minimal MM9 medium. This difference disappeared in iron-rich environment (minimal medium supplemented with iron and LB medium). 

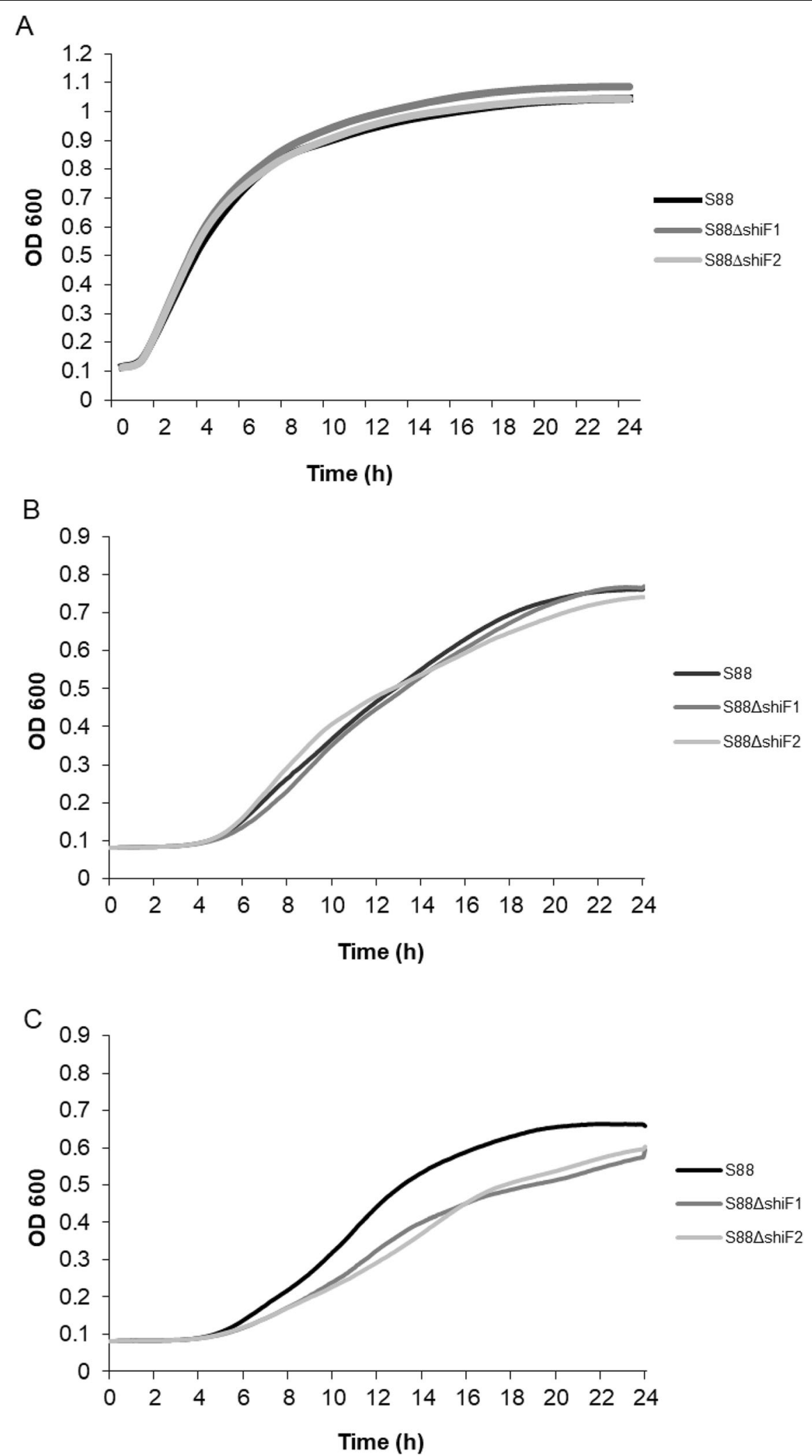

Fig. 1 Deletion of shiF reduces growth in iron-depleted medium. Strains were grown LB medium (a), in MM9 minimal medium with $20 \mu M$ iron (b) and $100 \mu \mathrm{M}$ of 2,2'-dipyridyl (c). Data presented are average of results from two (a) and five (b and $\mathbf{c}$ ) independent experiments 


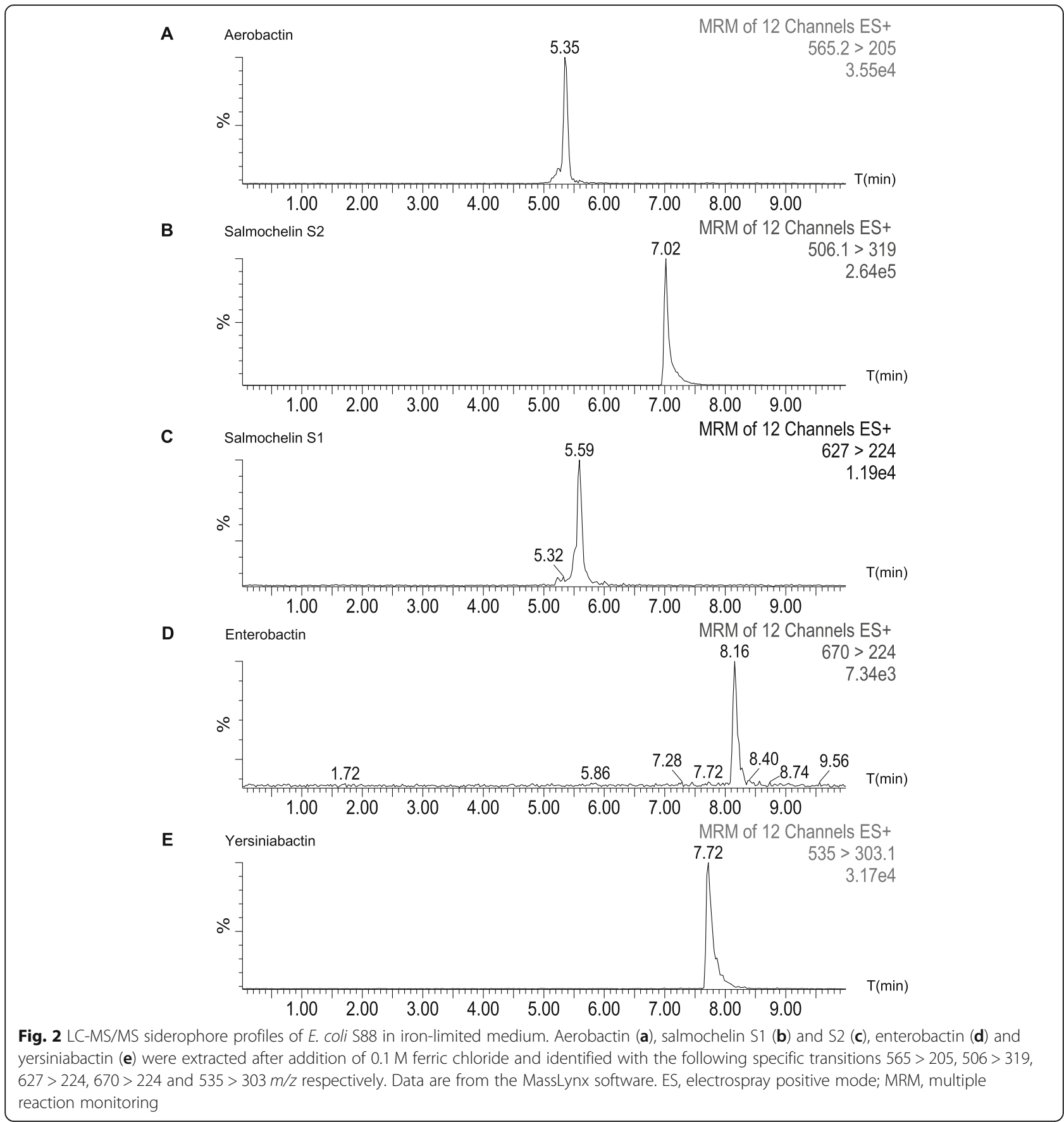

In silico analysis of shiF sequence reveals a strong similarity $(86 \%)$ with the membrane proteins belonging to the MFS suggesting a transporter role. This led us to propose two hypothetic functions for shiF: a role in aerobactin exportation or a role in aerobactin synthesis by importing lysin which is the aerobactin precursor. We analyzed siderophore production in the supernatant of S88 and S88 $\Delta$ shiF in iron-limited medium using LCMS/MS. There was no difference of enterobactin, yersiniabactin and salmochelin production but we found a significant decrease of aerobactin production for the shiF-deleted mutants. This confirmed that shiF is not essential but is involved in aerobactin synthesis or exportation. Analysis of intracellular contents of aerobactin showed no significant differences between wild type S88 and mutants but this result can't lead to conclude on the precise role of shiF.

Study of commensal strain ED1a reinforced the functional link between shiF and aerobactin. In this strain, shiF is adjacent to aerobactin operon but interrupted by 


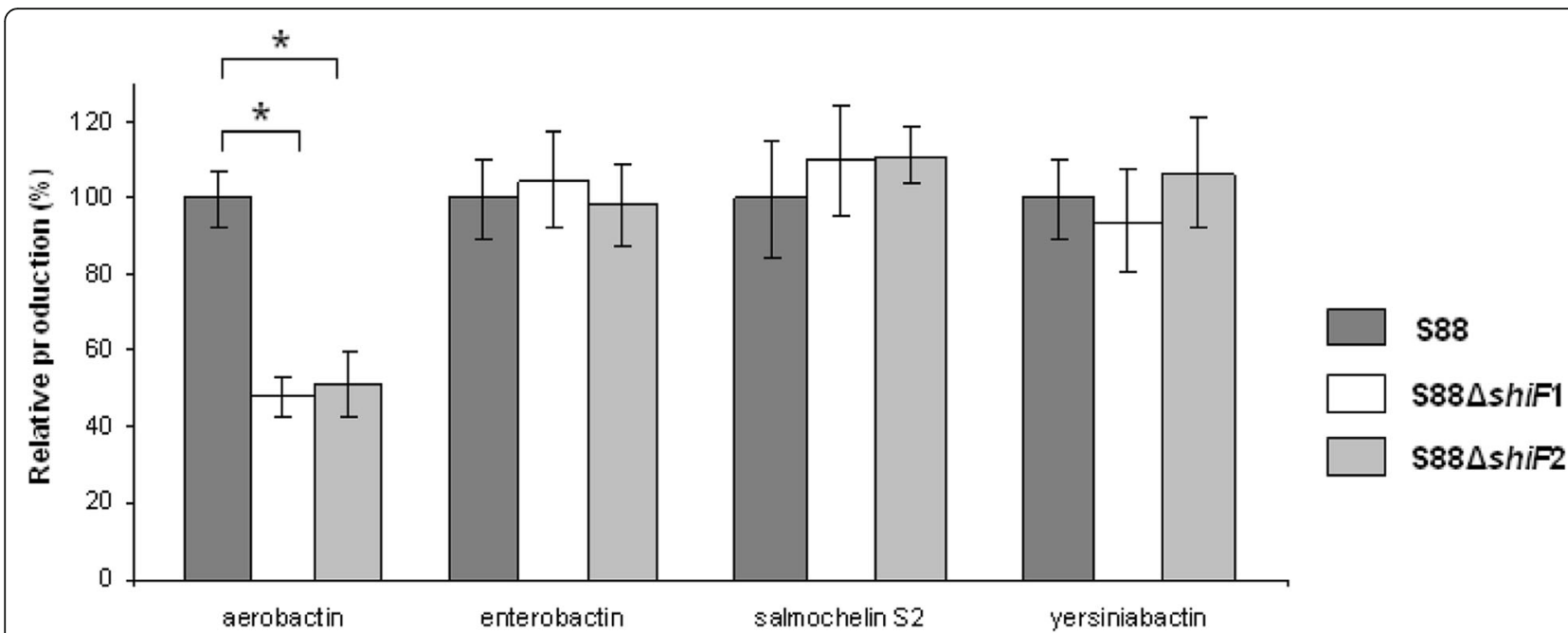

Fig. 3 Relative production of aerobactin, enterobactin, salmochelin S2 and yersiniabactin measured by LC-MS/MS. Production of aerobactin, enterobactin,

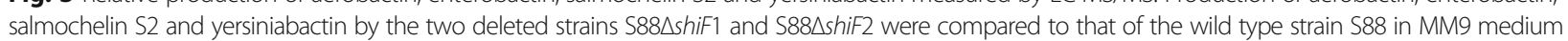
with $100 \mu \mathrm{M}$ of 2,2'-dipyridyl. Results are expressed in relation to the production of the wild type strain which represent 100\%. Data presented are means of 8 independent experiments and were compared using Mann-Whitney's test. Error bars represent the standard derivations. ${ }^{*} p<0.001$

a $10 \mathrm{~kb}$ insert and probably not functional. Cross-feeding assays and LC-MS/MS both revealed the inability of this strain to produce aerobactin despite the presence of the whole aerobactin operon. The strain harboring aerobactin and a truncated version of shiF is non-pathogenic and unable to produce aerobactin. The only NMEC strain harboring aerobactin but not shiF was iatrogenically inoculated, thus by-passing the bacteremia step that requires functional iron capture systems.

A limitation of this work was our inability to study our complemented shiF-deleted mutant because of its instability in minimal medium. Therefore, we studied two distinct mutants obtained from two separate mutagenesis experiments to reduce the risk of bias due to unwanted genetic events. Both mutants showed the same phenotype. Moreover, since our deletion primers were located inside shiF sequence and $32 \mathrm{pb}$ away from the start of aerobactin promoter a polar effect was unlikely [8] .

\section{Conclusions}

ShiF is physically and functionally linked to aerobactin. It procures an advantage during growth of E. coli S88 under limited iron conditions by acting as a booster for aerobactin secretion. However, we can't conclude on its precise role. ShiF like $s s b L$ appears to be an auxiliary virulence factor. This study reinforce the concept that virulence factors probably need acquisition of auxiliary genes to optimize their expression [4].

\section{Methods}

\section{In silico analysis}

50 E. coli genomes available in GenBank ${ }^{\odot}$ (http://ncbi. nlm.nih.gov/genbank/) were screened for presence of
shiF and aerobactin using BLAST ${ }^{\oplus}$ algorithm (http:// blast.ncbi.nlm.nih.gov/Blast.cgi)

\section{Bacterial strains and mutagenesis}

S88 is an NMEC strain representative of clone O45:K1:H7 belonging to phylogenetic subgroup $\mathrm{B} 2{ }_{1}$ and harbours the large plasmid pS88 which contains a functional aerobactin operon [2]. Strain S88 was isolated in our laboratory. Its genome accession number is CU928161. We deleted shiF from S88 using Datsenko and Wanner's method and the following primers Wanner-ShiF-1 (5'-ACAAATAAAC ACAATTA ATACATGGTGTTGAAACATTACATGTGTAGGCTGG AGCTGCT-3'), Wanner-ShiF-2 (5'-CACAGGACGTTATG CCGGCTGTGAGTACAACATCATAGCC.

CATATGAATATCCTCCTTAG-3') [9]. Two mu-

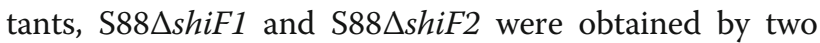
distinct mutagenesis experiments. One hundred wellcharacterized and epidemiologically unrelated ExPEC strains were screened to assess the distribution of shiF. These strains have been previously described and isolated in France between 2007 and 2012 [2, 10, 11].

Other strains used in this study were: $\mathrm{S} 88 \Delta \mathrm{pS} 88$ [2], $E$ coli $\mathrm{K}-12$ MG1655, E coli LG1522 [12] which is aerobactin dependent to grow under iron-limited conditions, the fully sequenced commensal E. coli strain ED1a and uropathogenic strain CFT073 which both harbor the aerobactin operon on their chromosome [13, 14]. Strains ED1-a, CFT073 and E coli K-12 MG1655 were obtained from Pr Erick Denamur head of our research team (Infection, Antimicrobials, Modelling, Evolution (IAME), University of Paris, France). Their genome accession numbers are respectively NC_011745, NC_004431 and NC_000913. Strain LG1522 was obtained from Pr Xavier 
Nassif (Institut Necker Enfants Malades (INEM) University of Paris, France).

\section{PCR prevalence of shiF in ExPEC strains}

The 100 ExPEC strains described above were screened by PCR to determine prevalence of shiF and its colocalization with aerobactin operon. Primers were based on the sequences of shiF and aerobactin operon in pS88 and are depicted in Fig. 4 and Table 1.

All PCRs were performed with Taq DNA polymerase (Qiagen) on an iCycler thermal cycler (Bio-Rad, Marnes La Coquette, France) as previously described [4]. Positive controls were the strains S88 and CFT073.

\section{Cross-feeding assays}

LB agar plate containing $200 \mu \mathrm{M}$ of the iron chelator 2,2dipyridyl, were seeded with strain E. coli LG1522 [12, 15]. The strains to be tested for aerobactin production were suspended in sterile water at an optical density at $600 \mathrm{~nm}$ $\left(\mathrm{OD}_{600}\right)$ of 1 and then spotted onto the agar. Plates were observed after $24 \mathrm{~h}$ incubation at $37^{\circ} \mathrm{C}$ for $24 \mathrm{~h}$ for growth of LG1522 strain around the spots.

\section{Growth assays}

Strains were routinely grown in LB (Sigma) or in MM9

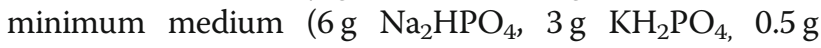
$\mathrm{NaCl}, 1 \mathrm{~g} \mathrm{NH} \mathrm{N}_{4} \mathrm{Cl}, 2 \mathrm{mM} \mathrm{MgSO} 4,0.1 \mathrm{mM} \mathrm{CaCl} 2$ per liter of water, pH 7.0) (Sigma-Aldrich) containing $20 \mathrm{mM}$ glucose and $0.5 \%$ of an amino-acid solution (Vaminolact ${ }^{\circ}$, Fresenius Kabi, Belgium) in a rotary shaker at $37^{\circ} \mathrm{C}$. Strains grown for $18 \mathrm{~h}$ in $\mathrm{LB}$ at $37^{\circ} \mathrm{C}$ with shaking were washed three times in fresh MM9 then diluted 1/10000 in fresh MM9 with $20 \mu \mathrm{M} \mathrm{FeSO}_{4}$ or $100 \mu \mathrm{M}$ of 2,2'-dipyridyl to ensure iron limitation. Strains were then grown with shaking for $24 \mathrm{~h}$ at $37^{\circ} \mathrm{C}$ in a multimode microplate reader (TECAN Infinite ${ }^{\circ} \mathrm{M} 200 \mathrm{PRO}$ ) and $\mathrm{OD}_{600}$ was measured every $5 \mathrm{~min}$ was used to determine cell density. Calculated maximum growth rates, maximum cell density and lag were used for statistical comparison of the strains [16].

\section{Extraction and LC-MS/MS analysis of siderophores from} culture supernatants and whole cells

Strains were suspended to an $\mathrm{OD}_{600}$ of 0.05 in $10 \mathrm{~mL}$ of MM9 with $100 \mu \mathrm{M}$ of 2,2'-dipyridyl and grown for $14 \mathrm{~h}$ at $37^{\circ} \mathrm{C}$ with shaking. After centrifugation, ferric chloride $0.1 \mathrm{M}$ was added to cell supernatants at a final concentration of $3.75 \mathrm{mM}$, as previously described [17]. After $15 \mathrm{~min}$ at room temperature, the precipitate was removed by centrifugation $(4000 \mathrm{~g}, 10 \mathrm{~min})$. One milliliter of supernatant was evaporated to dryness under a gentle stream of nitrogen at $45^{\circ} \mathrm{C}$ for $1 \mathrm{~h}$. Dry residues were resolvated in $100 \mu \mathrm{L}$ of $0.1 \%$ formic acid, and $5 \mu \mathrm{L}$ was directly injected for analysis. For intracellular extraction, bacterial pellets from initial centrifugation were washed with ice-cold MM9 at $+4{ }^{\circ} \mathrm{C}$, disrupted with 1.5 $\mathrm{mL}$ of ice-cold ethanol and sonication during $1 \mathrm{~min}$ and centrifuged again at $16000 \mathrm{~g}$ for $10 \mathrm{~min}$ [18]. One milliliter of the supernatants was then prepared as described above.

In order to obtain internal standards by isotopic labelling, strain S88 was grown in a rotary shaker for $14 \mathrm{~h}$ at $37^{\circ} \mathrm{C}$, in MM9 containing $20 \mathrm{mM}$ of ${ }^{13} \mathrm{C}$-glucose as the sole carbon source and $100 \mu \mathrm{M}$ of $2,2^{\prime}$-dipyridyl. ${ }^{13} \mathrm{C}$-labelled siderophores were extracted as described above and frozen for use as internal standards.

Siderophores were separated by reverse-phase liquid chromatography on a C18 column (Symmetry $2.1 \times 50$ $\mathrm{mm}, 3.5 \mu \mathrm{m}$, Waters) as previously described [4]. The liquid chromatograph (Separations module Alliance 2795, Waters) was coupled to a mass spectrometer with an electrospray ionization probe (Quattro micro ${ }^{\mathrm{TM}}$ Mass Spectrometer). Analyses were performed in positive ionization mode.

Strains to be compared were prepared together using same media, reagents and internal standard. The ${ }^{13} \mathrm{C}$-labeled internal standard was mixed to each culture supernatant (1:1) prior to siderophore extraction. Quantification was based on multiple reaction monitoring. Specific transition ions monitored from pseudomolecular ions to daughter ions used for

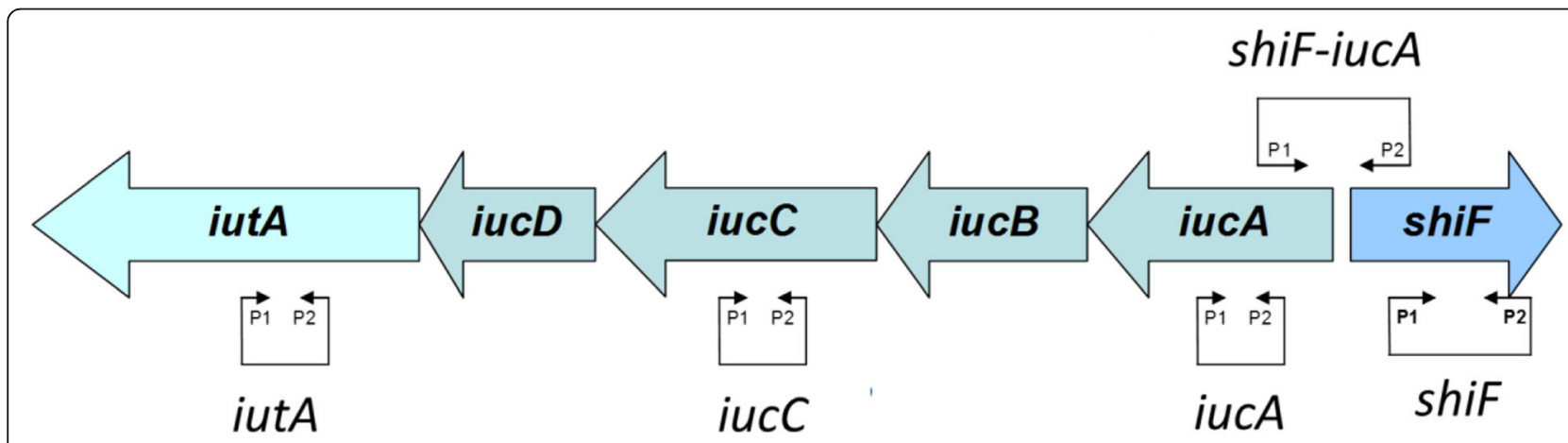

Fig. 4 Schematic representation of aerobactin operon and shiF. The large arrows represent the genes of the aerobactin operon and shiF lucA, iUCB, iUCC and iUCD encode the operon allowing aerobactin synthesis and iutA encodes aerobactin receptor. The small arrows annotated P1 and P2 represent the primers described in Table 1 and are located in front of their hybridizing sequences 
Table 1 Primers used for epidemiology of shif and aerobactin

\begin{tabular}{|c|c|c|c|}
\hline Name & 5'-3' Sequence & Gene & Product lenght \\
\hline shif-p.1 & GATCGAAGATACGCCCCAA & shiF & $1050 \mathrm{pb}$ \\
\hline shif-p.2 & CCATAGCCAAGTGTGTGACTG & & \\
\hline iutA-p.1 & CCGTAACCCGGGCTGTAGTA & iutA & $171 \mathrm{pb}$ \\
\hline iutA-p.2 & ACCACCTCCTTTGACGTGAG & & \\
\hline iucA-p.1 & AAGGGTCAACGATGGTGTTC & iucA & $185 \mathrm{pb}$ \\
\hline iucA-p.2 & ACAGCGAAGCGAATCTGATG & & \\
\hline iucC-p.1 & TGGACGCTGAAACCTGGCTTACGCAACTGT & iucC & $287 \mathrm{pb}$ \\
\hline iucC-p.2 & CACGAAGTGACCCGTCTGCAAATCATGGAT & & \\
\hline shif-iucA-p.1 & TCACGAATCAAGGCATTCAG & shif/ iucA ${ }^{a}$ & $400 \mathrm{pb}$ \\
\hline shif-iucA-p.2 & GATGGCAGAAACAGCATTGA & & \\
\hline
\end{tabular}

${ }^{\mathrm{a}}$ These primers are used to test the colocalization of shiF and iucA (Fig. 4)

detection and quantification of $[\mathrm{M}+\mathrm{H}]^{+}$aerobactin, $[\mathrm{M}+$ $\mathrm{H}]^{+}$enterobactin, $[\mathrm{M}-2 \mathrm{H}+\mathrm{Fe}(\mathrm{III})]^{+}$ferric-yersiniabactin, $[\mathrm{M}+\mathrm{H}]^{+}$salmochelin S2 (source-fragmented) and $[\mathrm{M}+\mathrm{H}]^{+}$ salmochelin S1 were 565 > 205, 670 > 224, $535>303,506>$ 319 and $627>224 \mathrm{~m} / z$, respectively. The respective ${ }^{13} \mathrm{C}$-labeled isotopes were $587>213,700>234,556>314,527>$ 332 and $653>234 \mathrm{~m} / z$ [17]. Using MassLynx software, each siderophore was quantified by determining the ratio of the area under the peak to the area of its corresponding ${ }^{13} \mathrm{C}$-labeled internal standard.

\section{Statistical analysis}

$P$ values were calculated using Mann Whitney U-test for five to eight replicate per experiment. $P$ values below 0.05 were considered statistically significant. Data are presented as mean \pm standard deviation.

\section{Supplementary information}

Supplementary information accompanies this paper at https://doi.org/10. 1186/s12866-019-1677-2

Additional file 1: Figure S1. Production of aerobactin analyzed by cross-feeding assay on LB agar plate containing $200 \mu \mathrm{M}$ of 2,2-dipyridyl

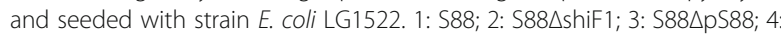

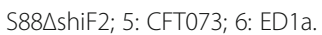

\section{Abbreviations}

ExPEC: Extra-intestinal pathogenic E. coli strains; LB: Lysogeny broth; LC-MS/ MS: Liquid chromatography-mass spectrometry; MFS: Major Facilitator Superfamily; NMEC: Neonatal meningitis E. coli; OD600: Optical density at 600 nm; ORF: Open reading frame; pS88: Plasmid carried by strain S88

\section{Acknowledgements}

We thank Pr Xavier Nassif for giving us the strain LG1522.

\section{Authors' contributions}

MG: made substantial contributions to conception and design, acquisition, analysis and interpretation of data. He has been involved in drafting the manuscript and revising it critically for important intellectual content. He has given final approval of the version to be published. PB: made substantial contributions to conception and design, acquisition, analysis and interpretation of data. He has been involved in drafting the manuscript and revising it critically for important intellectual content. He has given final approval of the version to be published. JFB: made substantial contributions to conception and design, acquisition, analysis and interpretation of data. He has been involved in revising the manuscript critically for important intellectual content. He has given final approval of the version to be published. DS: made substantial contributions to acquisition, analysis and interpretation of data. He has given final approval of the version to be published. CL: made substantial contributions to acquisition, analysis and interpretation of data. She has been involved in revising the manuscript critically for important intellectual content. She has given final approval of the version to be published. AB: made substantial contributions to acquisition, analysis and interpretation of data. He has been involved in revising the manuscript critically for important intellectual content. He has given final approval of the version to be published. SB: made substantial contributions to conception and design, acquisition, analysis and interpretation of data. He has been involved in drafting the manuscript and revising it critically for important intellectual content. He has given final approval of the version to be published.

\section{Funding}

This work was supported in part by the Fondation pour la Recherche Médicale (C.L.).

The funder had no role in the design, analysis or interpretation of data or in the writing of the manuscript.

\section{Availability of data and materials}

All data generated or analysed during this study are included in this published article.

The draft genome sequences of the $50 \mathrm{E}$. coli are available in GenBank ${ }^{\oplus}$ (http://ncbi.nlm.nih.gov/genbank). E. coli strains S88, ED1-a, CFT073 and K-12 MG1655 genome accession numbers are respectively CU928161, NC_011745, NC_004431 and NC_000913. The datasets used during the current study are available from the corresponding author upon request.

Ethics approval and consent to participate

Not applicable.

\section{Consent for publication}

Not applicable.

\section{Competing interests}

The authors declare that they have no competing interests.

\section{Author details}

'University Paris Diderot, Sorbonne Paris Cité, IAME, F-75018 Paris, France. ${ }^{2}$ Service de microbiologie, Hôpital Robert-Debré, AP-HP, 48 Bd Sérurier, 75019 Paris, France. ${ }^{3}$ Service de Biochimie-Hormonologie, AP-HP Hôpital Robert Debré, Paris, France. ${ }^{4}$ University Paris-Sud, Chatenay-Malabry, France. 
Received: 29 April 2019 Accepted: 5 December 2019

Published online: 17 December 2019

\section{References}

1. Bonacorsi S, Clermont O, Houdouin V, Cordevant C, Brahimi N, Marecat A, Tinsley C, Nassif X, Lange M, Bingen E. Molecular analysis and experimental virulence of French and north American Escherichia coli neonatal meningitis isolates: identification of a new virulent clone. J Infect Dis. 2003 187:1895-906.

2. Peigne C, Bidet P, Mahjoub-Messai F, Plainvert C, Barbe V, Medigue C,

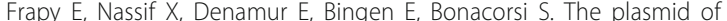
Escherichia coli strain $\mathrm{S} 88(\mathrm{O} 45: \mathrm{K} 1: \mathrm{H} 7)$ that causes neonatal meningitis is closely related to avian Pathogenic E. coli plasmids and is associated with high-level bacteremia in a neonatal rat meningitis model. Infect Immun. 2009;77:2272-84.

3. Lemaître $C$, Bidet $P$, Bingen $E$, Bonacorsi S. Transcriptional analysis of the Escherichia coli ColV-la plasmid pS88 during growth in human serum and urine. BMC Microbiol. 2012;12:115.

4. Lemaître $C$, Bidet $P$, Benoist J-F, Schlemmer D, Sobral E, d'Humières C, Bonacorsi S. The ssbL gene harbored by the ColV plasmid of an Escherichia coli neonatal meningitis strain is an auxiliary virulence factor boosting the production of siderophores through the shikimate pathway. J Bacteriol. 2014; 196:1343-9.

5. Moss JE, Cardozo TJ, Zychlinsky A, Groisman EA. The selC-associated SHI-2 pathogenicity island of Shigella flexneri. Mol Microbiol. 1999;33:74-83.

6. Garénaux A, Caza M, Dozois CM. The ins and outs of siderophore mediated iron uptake by extra-intestinal pathogenic Escherichia coli. Vet Microbiol. 2011:153:89-98.

7. Forman S, Nagiec MJ, Abney J, Perry RD, Fetherston JD. Analysis of the aerobactin and ferric hydroxamate uptake systems of Yersinia pestis. Microbiology. 2007;153:2332-41.

8. de Lorenzo V, Wee S, Herrero M, Neilands JB. Operator sequences of the aerobactin operon of plasmid ColV-K30 binding the ferric uptake regulation (fur) repressor. J Bacteriol. 1987;169:2624-30.

9. Datsenko KA, Wanner BL. One-step inactivation of chromosomal genes in Escherichia coli K-12 using PCR products. Proc Natl Acad Sci U S A. 2000;97: 6640-5.

10. Bocquet $N$, Sergent Alaoui A, Jais J-P, Gajdos V, Guigonis V, Lacour B, Chéron G. Randomized trial of oral versus sequential IV/oral antibiotic for acute pyelonephritis in children. Pediatrics. 2012;129:e269-75

11. Mahjoub-Messai F, Bidet P, Caro V, Diancourt L, Biran V, Aujard Y, Bingen E, Bonacorsi S. Escherichia coli isolates causing bacteremia via gut translocation and urinary tract infection in young infants exhibit different virulence genotypes. J Infect Dis. 2011;203:1844-9.

12. Carbonetti NH, Williams PH. A cluster of five genes specifying the aerobactin iron uptake system of plasmid ColV-K30. Infect Immun. 1984, 46:7-12.

13. Welch RA, Burland V, Plunkett G 3rd, Redford P, Roesch P, Rasko D, Buckles EL, Liou S-R, Boutin A, Hackett J, Stroud D, Mayhew GF, Rose DJ, Zhou S, Schwartz DC, Perna NT, Mobley HLT, Donnenberg MS, Blattner FR. Extensive mosaic structure revealed by the complete genome sequence of uropathogenic Escherichia coli. Proc Natl Acad Sci U S A. 2002;99:17020-4.

14. Touchon M, Hoede C, Tenaillon O, Barbe V, Baeriswyl S, Bidet P, Bingen $E_{\text {, }}$ Bonacorsi S, Bouchier C, Bouvet O, Calteau A, Chiapello H, Clermont O, Cruveiller S, Danchin A, Diard M, Dossat C, Karoui ME, Frapy E, Garry L, Ghigo JM, Gilles AM, Johnson J, Le Bouguénec C, Lescat M, Mangenot S, Martinez-Jéhanne V, Matic I, Nassif X, Oztas S, et al. Organised genome dynamics in the Escherichia coli species results in highly diverse adaptive paths. PLoS Genet. 2009:5:e1000344.

15. Nassif $X$, Mazert MC, Mounier J, Sansonetti PJ. Evaluation with an iuc::Tn10 mutant of the role of aerobactin production in the virulence of Shigella flexneri. Infect Immun. 1987:55:1963-9.

16. Bleibtreu A, Gros P-A, Laouenan C, Clermont O, Le Nagard H, Picard B, Tenaillon O, Denamur E. Fitness, stress resistance, and Extraintestinal virulence in Escherichia coli. Infect Immun. 2013;81:2733-42.

17. Henderson JP, Crowley JR, Pinkner JS, Walker JN, Tsukayama P, Stamm WE, Hooton TM, Hultgren SJ. Quantitative metabolomics reveals an epigenetic blueprint for iron acquisition in uropathogenic Escherichia coli. PLoS Pathog. 2009:5:e1000305.
18. Caza M, Lépine F, Dozois CM. Secretion, but not overall synthesis, of catecholate siderophores contributes to virulence of extraintestinal pathogenic Escherichia coli. Mol Microbiol. 2011;80:266-82.

\section{Publisher's Note}

Springer Nature remains neutral with regard to jurisdictional claims in published maps and institutional affiliations.
Ready to submit your research? Choose BMC and benefit from:

- fast, convenient online submission

- thorough peer review by experienced researchers in your field

- rapid publication on acceptance

- support for research data, including large and complex data types

- gold Open Access which fosters wider collaboration and increased citations

- maximum visibility for your research: over $100 \mathrm{M}$ website views per year

At $\mathrm{BMC}$, research is always in progress.

Learn more biomedcentral.com/submissions 